Research Paper

\title{
Quality Improvement of Artificial Cultivation of Anoectochilus Roxburghii
}

XiaOmin Xu

Shanghai Dianji University

1350 Ganlan, Lingang New City, Pudong District

Shanghai, China

E-mail: lygxxm@126.com

LEI SUN

Shanghai Dianji University

1350 Ganlan, Lingang New City, Pudong District

Shanghai, China

E-mail: sunlei@sdju.edu.cn

LIHUI SUI

Shanghai Dianji University

1350 Ganlan, Lingang New City, Pudong District

Shanghai, China

E-mail: suilh@sdju.edu.cn

YongXiang Cui

Shanghai Dianji University

1350 Ganlan, Lingang New City, Pudong District

Shanghai, China

E-mail: yxcui@sdju.edu.cn 


\section{About the author}

Xiaomin $\mathrm{Xu}$ is currently an associate professor of quality management engineering in School of Business, Shanghai Dianji University, China. She received her Bachelor of Engineering from China University of Mining and Technology, Master of Management from Nanjing University of Science and Technology, and Ph.D. in Management Science and Engineering from Beijing University of Science and Technology. Her research interests include logistics management, quality management and operation management. She published research articles in major academic journals within the field of engineering management in China, such as Statistics and Decision, Technical Economics and Management.

Lei Sun is a lecture of quality management engineering in School of Business, Shanghai Dianji University, China. He received his Bachelor of Management degree from Wuhan University of Science and Engineering, Master of Management from Dalian University of Technology, and Ph. D in Management Science and Engineering from Nueva Ecija University of Science and Technology in Philippines. His research interests include R\&D management, Quality Management and Lean Six Sigma Management.

Lihui Sui is currently a professor at School of Business, Shanghai Dianji University. She received her Bachelor of Engineering from the Department of Industry Management Engineering at Harbin University of Science and Technology in 1992, Master in Mechanical Manufacturing from Harbin University of Science and Technology in 1995 and Ph. D in Management Science and Engineering from Harbin Institute of Technology in 2007. Her research mainly focuses on quality management.

Youxiang Cui is currently a lecturer of quality management engineering at School of Business, Shanghai Dianji University, China. He received his bachelor degree in investigation and engineering from Chengdu Institute of Science and Technology, master degree in management science and engineering from Shandong University of Science and Technology, and Ph.D in management science and engineering From Tongji University. His research mainly focuses on quality management. 


\section{Abstract}

This study observes the artificial cultivation process for Anoectochilus roxburghii conducted by L. Company. The purpose of this study is to find out factors which are responsible for the cultivation of high quality Anoectochilus roxburghii through analysing the collected data using statistical methods. By investigating the different cultivation environments of Anoectochilus roxburghii, this study concludes that Anoectochilus roxburghi produced in Fujian Province has a better quality than those produced elsewhere. Furthermore, detailed analysis shows that the cultivation environment should meet the following conditions in order to obtain high-quality Anoectochilus roxburghii: medium MS, humus soil (600), the river sand (200) and perlite (200). Future work is required to prove the validity of this research result in the efficiency and quality improvement of Anoectochilus roxburghii cultivation.

Keywords: Anoectochilus Roxburghii, Artificial Cultivation, Rank Sum Test, Orthogonal Experiment Design

\section{Introduction}

Anoectochilus roxburghii (Orchidaceae family) is a native plant in some southern regions of China, such as Fujian, Guangdong, Hainan, Guangxi, Yunnan, Guizhou and Sichuan. Its distribution is also found in Taiwan. In recent years, Anoectochilus roxburghii has become much more well-known because of its special medicinal and ornamental value. Anoectochilus roxburghii produced in China is usually divided into two types: North Cordyceps sinensis and South Anoectochilus roxburghii (Li, 2001; Zhong, 1997). Through a long history, the Anoectochilus folk is more widely known by its laudatory names in China, such as Golden Grass, King of Medicine and Magic pill. In the Southeast Asia including Fujian and Taiwan areas, it has been used as a rare and precious medicinal herb traditionally for hundreds of years. Due to its great effect on the treatment of many diseases, such as pulmonary tuberculosis, lung cough, rheumatoid arthritis, infantile convulsion and injuries caused by snakes and insects (see Wu, Chuang, Yang and Lin, 2010; Refish, Talib, $\mathrm{Gu}, \mathrm{Fu}$ and $\mathrm{Yu}, 2016)$. The effect of Anoectochilus on the treatment of high blood pressure, diabetes and cancer has been proved recently, which makes it increasingly draw the attention from both researchers and professionals (Wu, Fen and He, 2008; Liu, Liu, Xiao, Zhou, Zhang and Li, 2013; Yu, Lian, Wu, Yu, Xie and $\mathrm{Wu}, 2015)$. But because of the over-collected and the loss of its suitable habitats, Anoectochilus is now in extinct condition (Zhang, Wang, Shao, Xua, Zhang and Li, 2015). The germination rate of the tiny seeds of Anoectochilus is very low and the less developed roots prevent the plants from growing fast in natural reproduction. Besides, the mountain areas are being developed fast for the economic benefits, the ecological conditions for the natural growth of Anoectochilus. Combined with the destruction problem caused by pest birds, wild Anoectochilus populations are declining sharply and on the verge of extinction (Wei, Xie, Qin and Chen, 2012; Xiao, Lai, Lin and Wu, 2014; Chen and Chen, 2014; Huang, 2015).Therefore, researchers in Fujian, Taiwan and Japan started conducting researches about its cultivation actively in recent years (Shao, 
Zhou, Hu, Zhang, Liu and Li, 2014; Wei, Xie, Qin and Chen, 2015 ${ }^{\mathrm{a})}$; Chen, 2015).

The artificial cultivation of Anoectochilus roxburghii is of crucial significance because it is the prerequisite for further research on its application for medicinal purposes (Zhang, 2004). This paper focuses on the issue of how to produce high-quality Anoectochilus roxburghii at relatively low cost.

\section{Previous Study on Product Quality and Anoectochilus}

Whenever the term product quality is discussed, price has often been used as an indicator for its measurement (Maynes, 1976). Deming (1982) defined quality as "a predictable degree of uniformity and dependability, at low cost and suited to the market." "Differences in product quality reflect differences in the quantity of some ingredient or attribute possessed by a product" (Garvin, 1984). Therefore, Product quality tends to be threatened when there is an excessive control of cost in the production process, because the producers might have to replace the expensive ingredients with affordable materials (Leffler, 1982; Lancaster, 1971). The value of Anoectochilus is mainly embodied through its medicinal ingredients, namely, polysaccharide, flavones and alkaloids, which are significant indicators for its quality. How to achieve high-quality Anoectochilus at low cost to meet the increasing market demand is a challenge for advanced research in artificial cultivation.

There have been many attempts in artificial cultivation of Anoectochilus. Studies report progress in Japan (Akihisa, 2007), Pakistan (Ayub, 2008), India (Anilkumar, 2007) and Nepal (Pandey, 2006). The artificial cultivation techniques are believed to have been applied by more than 100 countries and areas in the world. Artificial cultivation techniques can greatly shorten the growth period of Anoectochilus and overcome the adverse effects on the plant itself caused by continuous planting on the same pieces of land. Culture solution and fertilizers are widely used to achieve high yield, good quality and less polluted Anoectochilus (Sasaki, 1998). Tissue culture technique has also been applied to the artificial cultivation of Anoectochilus roxburghii by many researchers (Chen, Lin, Zou and Lin, 2008; Piao, Chakarabarty, Hahn and Paek, 2003; Liu, Wei and Cen, 2009; Cen, Wei and Liu, 2009; Kan, Zhang and Li, 2009; Liu and Wang, 2009). Although the technique has been able to achieve some success, a decisive breakthrough is still to be expected. Anoectochilus has long been in short supply or even out of stock both at the domestic market in China and abroad (Zhao, 2008). Therefore, it is of crucial significance to conduct detailed investigation on the ecological habit and quality control of Anoectochilus roxburghii in order to discover effective methods to increase its amount of production and improve its quality as well.

Technical management level has reached an unprecedented increase in the scope of business activities, realized intensive technicalization and industrialization, and achieved the goal of good quality, high yield, high efficiency and low cost in regional production (Chen, Lin, Zou and Lin, 2008; Zhao and Guo, 2011; Wei, Xie, Qin and Chen, 2015 ${ }^{\mathrm{b})}$ ). According to Wang and Hao (2003), three steps are needed for quality analysis before any quality-related problems can be solved: hazard analysis, cause analysis and problem solution analysis. Sound scientific methods, such as statistic techniques, should be employed to analyze the exact cause of the quality-related problems on a knowledge basis. Decision-making process should be established on the result of quantitative analysis and systematic testing (Jia, 2014). You, Zhou and Wu et al. (2014) further 
explains in detailed the indispensable practice for quality analysis, such as pareto diagram, cause and effect diagram, histgram, variance analysis, signed rank sum test, hypothesis testing and orthogonal experimental design. Thus, with the help of such statistical analysis techniques, high-standard quality management can be achieved for the artificial cultivation of Anoectochilus roxburghii. The improvement of its output quality will expand its scope in both application and business.

This paper analyzes the artificial cultivation techniques of Anoectochilus roxburghii from the perspective of quality analysis and compares the Anoectochilus production process in Fujian and Taiwan. The purpose of this study is to put forward corresponding countermeasures and suggestions on how to boost the quality of the output.

\section{Model Specification and Methodology}

\section{Rank Sum Test}

The rank sum test is also commonly called the Mann-Whitney rank sum test or simply the Mann-Whitney test. It is "a nonparametric alternative to the two sample t-test which is based solely on the order in which the observations from the two samples fall" (Wild, 1997). When the populations are non-normal, particularly for small samples, then the t-test may not be valid as a standard test for testing that the difference between population means for two non-paired samples are equal. The rank sum test is an alternative that can be applied when distributional assumptions are suspect. It is one of the significant testing methods for data with uncertain population distribution.

In this study, Anoectochilus produced in Fujian and Taiwan are selected as samples and the differences between these two varieties are to be discovered. Because the distribution of collected data is not known, rank sum test is used for data analysis.

\section{Orthogonal Experimental Design Method}

Orthogonal design is "a powerful method for comparative effectiveness research with multiple interventions" (Zurovac and Brown, 2012) and has been widely applied to research within the management and manufacturing fields. It is a quantitative analysis method which can find out the influence degree of each individual factor on the problem, so as to accurately determine the weak factors among a whole and provides reliable evidence for further quality analysis. In this study, orthogonal experimental design method is used to discover factors causing problems for the artificial cultivation of Anoectochilus roxburghii and offer suggestions on the improvement of its quality management. 


\section{Comparative Analysis of Anoectochilus roxburghii using Rank Sume}

\section{Test}

There are strict environmental regulations for the artificial cultivation of high-quality Anoectochilus roxburghii. The temperature requirement is $15^{\circ} \mathrm{C}-30^{\circ} \mathrm{C}$, and humidity should be controlled between $60 \%$ and $80 \%$. Generally speaking, light intensity should be adjusted within the range of $3000-3500 \mathrm{~lx}$. The experiment was conducted with the support of L. Company located in Zhejiang, China. Its Greenhouse Cultivation Lab provides an ideal environment for the cultivation of Anoectochilus roxburghii. The temperature, humidity and light intensity was kept at a most favorable level as described above with utmost care and attention so that the plant was able to achieve and sustain desirable growth. Two culture disks were set up in the lab, with one labeled as Fujian Anoectochilus roxburghii and the other labeled as Taiwan Anoectochilus roxburghii. There are 200 plants in each of the disks. The whole cultivation process lasts for 90 days. The end products are collected after the cultivation period. In order to collect samples for this study with no discrimination, ten plants were randomly selected from each disk and weighed using a special electronic scale after being washed and dried. The net weight data of the collected Anoectochilus roxburghii are obtained as in table 1.

Table 1 Net Weight of Anoectochilus roxburghii

\begin{tabular}{lllllllllll}
\hline Districts & \multicolumn{10}{c}{ Net weight $(10 \mathrm{mg})$} \\
\hline Fujian & 92 & 78 & 76 & 87 & 89 & 94 & 90 & 88 & 85 & 88 \\
Taiwan & 65 & 69 & 86 & 80 & 68 & 63 & 76 & 82 & 75 & 66 \\
\hline
\end{tabular}

The rank sum test for the data in table 1 is conducted. The rank table (Table 2) is achieved according to the data size order. $A$ stands for Fujian Anoectochilus roxburghii, and $B$ stands for Taiwan Anoectochilus roxburghii.

In this study, $n_{A}=n_{b}=10$ is a prerequisite for the rank sum test. The sum of ranks $\left(T_{A}\right)$ of group A is $T_{A}=7.5+9+12+14+15.5 \times 2+17+18+19+20=147.5$. The sum of ranks $\left(T_{B}\right)$ of group B data is $T_{B}=1+2+3+4+5+6+7.5+10+11+13=61.5$. The statistic is $Z=\min (A, B)=61.5$.

Given that the significant level of $\alpha$ is $0.05, T_{A}=83$ and $T_{B}=127$ can be obtained through checking the rank sum test table. Therefore, it is included that if $T_{A} \leq T \leq T_{B}$, the null hypothesis $H_{0}$ is acceptable (There is no significant difference between $A$ and $B$ ) is accepted; if $T<T_{A}$ or $T>T_{B}$, the null hypothesis $H_{0}$ is rejected (There is a significant difference between the quality of Anoectochilus roxburghii produced in Fujian and Taiwan despite the same cultivation conditions. The quality of Fujian Anoectochilus roxburghii is better than that of Taiwan. 
Table 2 Net Weight Order of Anoectochilus roxburghii

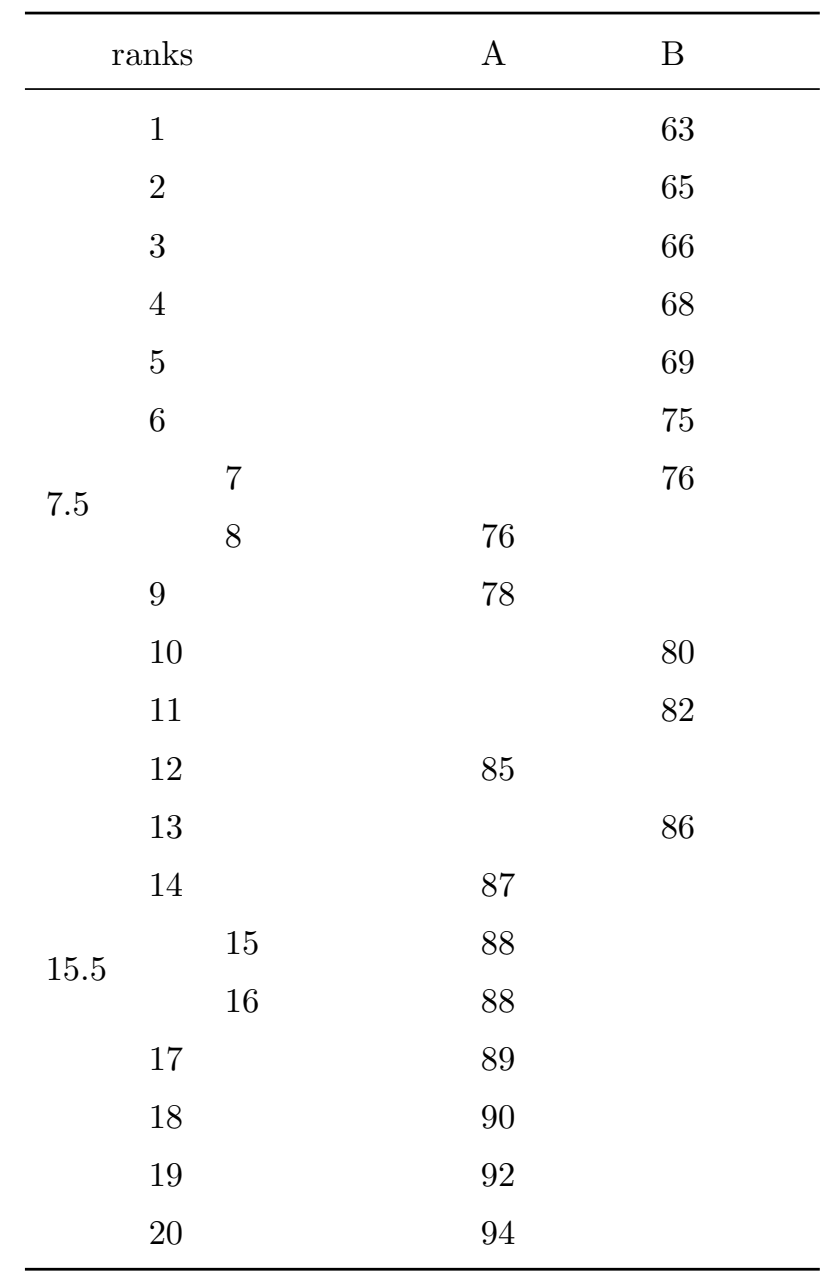

\section{Improvement of The Output Quality of Fujian Anoectochilus}

\section{Roxburghii}

The valuable ingredients of Anoectochilus roxburghii for medicinal use are polysaccharide, flavones and alkaloids. The result of the rank sum test suggests that more effective ingredients are contained in Fujian Anoectochilus roxburghii than Taiwan Anoectochilus roxburghii. In order to promote the quality of Fujian Anoectochilus roxburghii and obtain the strongest seeding for future artificial cultivation, an experiment is conducted in this study by mixing up humus soil, river sand and perlite with three mediums in different amounts - MS, AR and SE. The purpose of this is to create a most fertile and productive soil for the cultivation of Anoectochilus roxburghii and confirm the most favorable conditions for cultivation so that the effective ingredients of the produced Anoectochilus roxburghii could reach the maximum level. The analysis process consists of the following three steps: 
- Step 1: Set up a clear objective for the test and determine evaluation index.

As explained above, the objective is to discover the most suitable combination of the environmental factors, which affects the growth of Anoectochilus roxburghii and enhance the quality of the output. Therefore, the most significant evaluation index is the amount of effective ingredients included in the final produced Anoectochilus roxburghii.

- Step 2: Select factors for the different levels.

Four factors and three levels are identified according to the theory and practice for the experiment as in Table 3 .

Table 3 Factor Level Table

\begin{tabular}{lllll}
\hline \multirow{2}{*}{ Levels } & \multicolumn{4}{c}{ Factor } \\
\cline { 2 - 5 } & Mediums & Humus soil & River sand & Perlite \\
\hline 1 & MS & 400 & 300 & 300 \\
2 & AR & 500 & 300 & 200 \\
3 & SE & 600 & 200 & 200 \\
\hline
\end{tabular}

- Step 3: Create the appropriate orthogonal table.

Four factors and three levels from the directions for the orthogonal table $L_{9}\left(3^{4}\right)$. Fujian Anoectochilus roxburghii was cultivated for a 90-day grows cycle in a most desirable cultivation environment as described above. After thecultivation, the collected sample plants were sent to an authoritative inspection and quarantine orgnization for measurement of the three crucial ingredients - flavonoids, alkaloids and polysaccharide. Table 4 shows the detailed data.

Table 4 Ingredients of Fujian Anoectochilus roxburghii

\begin{tabular}{ccccccccc}
\hline Levels & Mediums & $\mathrm{A}$ & $\mathrm{B}$ & $\mathrm{C}$ & $\mathrm{D}$ & $\mathrm{E}$ & $\mathrm{F}$ & $\operatorname{Total}(\mathrm{D}+\mathrm{E}+\mathrm{F})$ \\
\hline 1 & $\mathrm{MS}$ & 400 & 300 & 300 & 41 & 38 & 25 & 104 \\
2 & $\mathrm{MS}$ & 500 & 300 & 200 & 42 & 32 & 27 & 101 \\
3 & $\mathrm{MS}$ & 600 & 200 & 200 & 53 & 39 & 28 & 120 \\
4 & $\mathrm{AR}$ & 400 & 300 & 300 & 38 & 29 & 22 & 89 \\
5 & $\mathrm{AR}$ & 500 & 300 & 200 & 31 & 35 & 17 & 83 \\
6 & $\mathrm{AR}$ & 600 & 200 & 200 & 39 & 30 & 25 & 94 \\
7 & $\mathrm{SE}$ & 400 & 300 & 300 & 41 & 26 & 18 & 85 \\
8 & $\mathrm{SE}$ & 500 & 300 & 200 & 31 & 30 & 29 & 90 \\
9 & $\mathrm{SE}$ & 600 & 200 & 200 & 45 & 25 & 22 & 92 \\
\hline
\end{tabular}

Note: A: Humus soil, B: River sand, C: Perlite, D: Polysaccharide, E: Flavones, F: Alkaloids 
Table 5 Homogeneity Test of Variances

\begin{tabular}{llll}
\hline & \multicolumn{3}{l}{$\mathrm{N}$} \\
\hline Medium & AR & 9 & \\
& MS & 9 & \\
& SE & 9 & \\
Additive & A & 9 & \\
& B & 9 & \\
& $\mathrm{C}$ & 9 & \\
\hline \hline F & $\mathrm{df}_{1}$ & $\mathrm{df}_{2}$ & Sig. \\
1.611 & 8 & 18 & 0.190 \\
\hline
\end{tabular}

According to the evaluation criteria of L. Company, the three important ingredients in Anoectochilus roxburghii, polysaccharide, flavonoid and alkaloid, should not go below $50 \pm 3 \mathrm{mg}$ and $30 \pm 3 \mathrm{mg}$, respectively. The higher the amount for each ingredient, the better of the quality. That is, Anoectochilus roxburghii with the highest amount of polysaccharide, flavonoid and alkaloid is considered the top level in the area of quality. Through comparison of the total amount of effective ingredients in table 4, conclusion is made that the best-quality Fujian Anoectochilus roxburghii is available by employing the medium (MS) with humus soil(600), river sand (200) and perlite (200).

- Step 4: Verify the result

SPSS 16 software is used to verify the result through homogeneity test of variances. The output is shown as in table 5 .

The homogeneity test of variances for univariate analysis is indicated as in table 6 . According to table 6 , the sig. value is 0.190 , more than the significant level 0.05 . This shows that the data meets the homogeneity of variance assumption. The effect test results are shown in table 6 .

The corrected Model in Table 6 is the test of the variance analysis model. All factors in the model including the medium and the additive (A, B, C) have no significant effects on the cultivation output of Fujian Anoectochilus roxburghii. The value of significant level for the Corrected Model is close to zero, which implies that the proposed model is a significant fit for the data. The Intercept in Table 6 is a constant item in the model. It has no practical significance in the analysis and should not be taken into account. The significance levels of the Medium and the Addictive items in Table 6 are indicative because both sig. values are less than $0.05(\alpha)$. This means that both the medium and the addictive (A, B and C) have a significant effect on the quality of the cultivated Anoectochilus roxburghii. The sig. value of the fifth item (Medium * Additive) is 0.624 , which is much greater than $0.05(\alpha)$.

This implies that the interaction of medium and additive is not significant in this study. The multiple decision coefficient $R$-Squared is 0.795 . This shows that the factor medium explains $79.5 \%$ 
Table 6 Effect Test Results

\begin{tabular}{crrrrr}
\hline Sources & Type III Sum of Squares & df & Mean Square & F & Sig. \\
\hline Corrected Model & 159.333 & 8 & 191.167 & 8.748 & 0.000 \\
Intercept & 27265.333 & 1 & 27265.333 & $1.248 \mathrm{E} 3$ & 0.000 \\
Medium & 253.556 & 2 & 126.778 & 5.802 & 0.001 \\
Additive & 1217.556 & 2 & 608.778 & 27.859 & 0.000 \\
Medium * Additive & 58.222 & 4 & 14.556 & 0.666 & 0.624 \\
Error & 393.333 & 18 & 21.852 & & \\
Total & 29188.000 & 27 & & & \\
Corrected Total & 1922.667 & 26 & & & \\
\hline$R-$ Squared $=0.795($ AdjustedR - Squared $=0.704)$ & & &
\end{tabular}

Table 7 Multiple Comparison of Medium and Addictive

\begin{tabular}{|c|c|c|c|c|c|c|}
\hline \multirow{2}{*}{ (I) } & \multirow{2}{*}{$(\mathrm{J})$} & \multirow{2}{*}{$\begin{array}{l}\text { Mean Difference } \\
\qquad(\mathrm{I}-\mathrm{J})\end{array}$} & \multirow{2}{*}{ Std.Error } & \multirow{2}{*}{ Sig. } & \multicolumn{2}{|c|}{ 95\% Confidence Interval } \\
\hline & & & & & Lower Bound & Upper Bound \\
\hline \multirow[t]{2}{*}{$\mathrm{A}$} & B & $8.5556^{*}$ & 2.20363 & 0.001 & 3.9259 & 13.1852 \\
\hline & $\mathrm{C}$ & $16.4444^{*}$ & 2.20363 & 0.000 & 11.8148 & 21.0741 \\
\hline \multirow[t]{2}{*}{ B } & A & $-8.5556^{*}$ & 2.20363 & 0.001 & -13.1852 & -3.9259 \\
\hline & $\mathrm{C}$ & $7.8889^{*}$ & 2.20363 & 0.002 & 3.2592 & 12.5185 \\
\hline \multirow[t]{2}{*}{$\mathrm{C}$} & A & $-16.4444^{*}$ & 2.20363 & 0.000 & -21.0741 & -11.8148 \\
\hline & B & $-7.8889^{*}$ & 2.20363 & 0.002 & -12.5185 & -3.2592 \\
\hline
\end{tabular}

*The mean difference is significant at the 0.05 level.

Table 8 Estimates of the Marginal

\begin{tabular}{cccc}
\hline \multirow{2}{*}{ Mean } & \multirow{2}{*}{ Std.Error } & \multicolumn{2}{c}{$95 \%$ Confidence Interval } \\
\cline { 3 - 4 } & & Lower Bound & Upper Bound \\
\hline 31.778 & 0.900 & 29.888 & 33.668 \\
\hline
\end{tabular}

relies on other random factors.

Multiple comparison results of these two items, medium and additive, are presented in Table 7 . Table 8 shows the marginal estimates of the quality of Fujian Anoectochilus roxburghii. 


\section{Conclusions}

In order to find out the most productive environment for the cultivation of Anoectochilus roxburghii, this study carries out a comparative study to investigate the quality of Fujian Anoectochilus roxburghii and Taiwan Anoectochilus roxburghii, which were cultivated under the same conditions, with controled temperature, humidity and light intensity. The rank sum test shows that there is significant difference between these two items in term of output quality. Fujian Anoectochilus roxburghii has more effective ingredients than the Taiwan product. This establishes the foundation for further study on how to improve the quality of the Fujian Anoectochilus roxburghii. Different combinations of mediums and additives are employed for the cultivation and the quality of Anoectochilus roxburghii cultivated under different condition is investigated. The combination of medium MS, humus (600), river sand (200), perlite (200) proves to be the constituents for the best growth environment of Anoectochilus roxburghii based on data of repetitive experiments.

The statistical analysis achieves good results with a sound argument. However, there are limitations in this study due to the relatively small size of samples. More efforts are needed to modify the cultivation conditions for high-quality Anoectochilus growth. More practical data is needed to validate the effectiveness of the mixture of medium AR and SE with different additives - humus soil, river sand and perlite for the cultivation of Anoectochilus roxburghii. The potential effects on the interaction of three mediums (MS, AR and SE) and addictives (humus soil, river sand and perlite) are to be further explored by using more sample data. In Anoectochilus roxburghii. Both the methods and the construction put forward in this study will lead to the construction of a through framework of practice for the cultivation of high-quality Anoectochilus roxburghii.

\section{Acknowledgment}

This study is supported by Academic Discipline Project of Shanghai Dianji University. Project Number :16YSXK02.

\section{References}

Akihisa, T., Nakamura, Y., Tokuda, H., Uchiyama, E., Suzuki, T.; Kimura, Y., Uchikura, K. and Nishino, H., 2007.

"Triterpene Acids from Poria Cocos and Their Anti-tumor-promoting Effects", Journal of Natural Products, Vol. 70, Issue 6, pp. 948-953.

Anilkumar, A. S., Beena, J. S., Suharban, M. and Harikrishnan Nair, K., 2007. "Medicinal Trees for

Biofencing", Journal of Natural Products, Vol. 70, Issue 6, pp. 948-953.

Ayub, M., Nadeem, M. A., Tanveer, A., Tahir, M., Saqib, M. T. Y. and Nawaz, R., 2008. "Effect of Different Sowing Methods and Times on the Growth and Yield of Fennel (Foeniculum Vulgare Mill)", Pakistan Journal of Botany, Vol. 40, Issue 1, pp. 259-264.

Cen, X., Wei, P. and Liu, F., 2009. "Effect of Plant Grows Regulators on Proliferation and Rootage of 
Anoectochilus formosaus Test-tube Plantlets", Journal of Guangxi Agriculture, Vol. 24, Issue 2, pp. 19-21.

Chen, H., 2015. "Cultivation Techniques of Fujian Anoectochilus Roxburghii", Fujian Agricultural Science and Technology, Issue 10, pp.61-63.

Chen, H. and Chen, S., 2014. "Achievements and Suggestion on the Implementation of 'Five New' Integrated Extension and Demonstration Project of Anoectochilus Roxburghii Industry in Nanjing County", Fujian Agricultural Science and Technology, Issue 1, pp. 79-81.

Chen, Y., Lin, Y., Zou, H. and Lin, J., 2008. "Quick Heavy Technique in Tissue Culture of Anoectochilus roxburghii and Anoectochilus Formosaus", Modern Horticulture, Vol. 10, pp. 9-12.

Deming, W. E., 1982. Quality Productivity and Competitive Position. Massachusetts, USA: Massachusetts Institute Technology.

Garvin, D. A., 1984. What Does "Product Quality" Really Mean? MIT Sloan Management Review, http://sloanreview.mit.edu/article/what-does-product-quality-really-mean/, retrieved Oct. $12,2016$.

Huang, D., 2015. "Pollution-free Cultivation Technology for Anoectochilus Roxburghii in Fujian with Greenhouse ", Fujian Agricultural Science and Technology, Issue 5, pp. 51-52.

Jia, J., 2014. Statistics (The Fifth Edition). Beijing, China: China Renmin University Press.

Kan, S., Zhang, M. and Li, H., 2009. "Induction of Adventitious Buts of Anoectochilus roxburghii", Journal of Anhui Agricultural Science, Vol. 37, Issue 3, pp. 981-982.

Lancaster, K., 1971. Consumer Demand: A New Approach. New York, USA: Columbia University Press.

Leffler, K. B., 1982. Ambiguous Changes in Product Quality, The American Economic Review, Vol. 72, No. 5, pp. 956-967

Li, J., 2001. "Anoectochilus Formosaus", Taiwan Agricultural Research, Issue 2, p. 42.

Liu, F., Wei, P. and Cen, X., 2009. "Effects of Explants and Basic Media on Clustered Shoots Induction of Anoectochilus formosanus", Northern Horticulture, Issue 4, pp. 103-104.

Liu, W. and Wang, N., 2009. "Screening on Multiplication Medium of Tissue Culture for Anoectochus roxburghii", Journal of Anhui Agricultural Science, Vol. 37, Issue 4, pp. 1475-1476.

Liu, Z. L., Liu, Q., Xiao, B., Zhou, J., Zhang, J. G. and Li, Y., 2013. "The Vascular Protective Properties of Kinsenoside Isolated From Anoectochilus Roxburghii Under High Glucose Condition", Fitoterapia, No. 86, pp. 163-170. DOI: 10.1016/j.fitote.2013.03.006.

Maynes, E. S., 1976. The Concept and Measurement of Product Quality. In Terleckyj, N. E. (ed), Household Production and Consumption. Massachusetts, USA: The National Bureau of Economic Research.

Pandey, Y. R., Karki, T. B. and Paudel, G. P., 2006. "Ginger Integration in Maize Based Cropping System for Increased Income in the Hills of Nepal", Nepal Journal of Science and Technology, Vol. 7, pp. 19-22.

Piao, X. C., Chakarabarty, D., Hahn E. J. and Paek, K. Y., 2003. "A Simple Method for Mass Production of Potato Microtubers Using a Bioreactor System", Current Science, Issue 84, pp. 1129-1132.

Refish, N. M. R., Talib, A. J., Gu, J. W., Fu, C. H. and Yu, L. J., 2016. Promoting Role of Bacillus Subtilis BS87 on the Growth and Content of Some Natural Products in the Medicinal Plants Anoectochilus 
Roxburghii and A. Formosanus. Advances in Life Sciences, Vol. 6, No. 2, pp. 31-38.

Sasaki, A., 1988. "The Present State and Problems in Hydroponic Culture (3) Composition of Nutrient Solution and Two to Three Kinds of Management Techniques", Agriculture and Horticulture, Issues 57, pp. 677-682.

Shao, Q., Zhou, A., Hu, R., Zhang, Y., Liu, T. and Li, M., 2014. "Influence of Seeding Grade on Plant Growth, Yield and Quality of Anoectochilus Roxburghii", China Journal of Chinese Material Media, Issue 5, pp. 785-789.

Xiao, K., Lai, R., Lin, R and Wu, G., 2014. "Effect of Different Culture Conditions on Main Chemical Composition of Anoectochilus Roxburghii", Journal of Chinese Medicinal Materials, Issue 4, pp. 553-556.

Yu, C. W., Lian, Q., Wu, K. C., Yu, S. H., Xie, L. Y. and Wu, Z. J., 2015. "The Complete Chloroplast Genome Sequence of Anoectochilus Roxburghii", Mitochondrial DNA Part A, Vol. 27, Issue 4, pp. 1-2.

Wang, Y. and Hao, F., 2003. Quality Analysis, Quality Improvement and Statistical Technology. Beijing, China: China Metrology Press.

Wei, C., Xie, Y., Qin, J. and Chen, Q., 2012. "High-yielding and High-quality Cultivation Technique for Anoectochilus Roxburghii", Fujian Agricultural Science and Technology, Issue 10, pp. 31-33.

Wei, C., Xie, Y., Qin, J. and Chen, Q., 2015 a) . "Effect of Fertilizations on Yield and Quality of Anoectochilus Roxburghii", Fujian Journal of Agricultural Sciences, Issue 8, pp.793-796.

Wei, C., Xie, Y., Qin, J. and Chen, Q., 2015 $5^{\mathrm{b}}$. "Effect of Light Intensity on the Growth and Yield of Anoectochilus roxburghii", Northern Horticulture, Issue 12, pp.139-141.

Wild, C., 1997. The Wilcoxon Rank-Sum Test. Retrieved on Oct. 10, 2016, https://www.stat. auckland. ac.nz/ wild/ChanceEnc/Ch10. wilcoxon.pdf.

Wu, C., Fen, Y. and He, M., 2008. "Research on Anoectochilus Seed Development", China's Wild Plant Resources, Vol. 27, Issue 2, pp. 47-50.

Wu, J. B., Chuang, H. R., Yang, L. C. and Lin, W. C., 2010 "A standardized Aqueous Extract of Anoectochilus formosanus Ameliorated Thioacetamide-Induced Liver Fibrosis in Mice: The Role of Kupffer Cells", Bioscience, Biotechnology, and Biochemistry, Vol. 74, Issue 4, pp. 781-787. Oxford, UK:Taylor \& Francis.

You, J., Zhou, W., Wu, X. and Shao, L., 2014. Quality Management (The Third Edition). Beijing, China: Science Press.

Zhang, G., 2004. "The Present Situation and Development Prospects of the Soilless Cultivation Technology", Gansu Agricultural Science and Technology, Issue 2, pp. 6-8.

Zhang, A., Wang, H., Shao, Q., Xua, M., Zhang, W. and Li, M., 2015. "Large Scale in Vitro Propagation of Anoectochilus Roxburghii for Commercial Application: Pharmaceutically Important and Ornamental Plant", Industrial Crops and Products, Vol. 70, pp. 158-162. Holland, Elsevier.

Zhao, Y., 2008. "Preliminary Study on Utilization Value of Anoectochilus Roxburghii and Its Cultivation Technique under Forest", Forest Inventory and Planning, Vol. 33, Issue 3, pp. 61-63.

Zhao, Y. and Guo, S., 2011. "Experimental Study of Improving the Toot Growth of Anoectochilus Formosanus and Anoectochilus roxburghii", Liaoning Agricultural Sciences, Issue 5, pp. 51-52. 
Zhong, C., 1997. "Medicinal Application Value and Development of Anoectochilus", Journal of Southern Agriculture, Issue 2, pp.102-104.

Zurovac, J. and Brown, R., 2012. Orthogonal Design: A Powerful Method for Comparative Effectiveness Research with Multiple Interventions. Retrieved on Oct. 14, 2016, https://www.mathematica-mpr.com/ search?keyword=0rthogonal Design: A Powerful Method for Comparative, Washington, USA: Mathematica Policy Research, Inc. 